\title{
Effects of Mole Fraction Variations and Scaling on Total Variability in InGaAs MOSFETs
}

\author{
Nicolò Zagni ${ }^{\dagger}$, Francesco Maria Puglisi, Paolo Pavan \\ Dipartimento di Ingegneria "Enzo Ferrari" (DIEF) \\ Università degli Studi di Modena e Reggio Emilia \\ 41125, Modena (MO), Italy \\ †nicolo.zagni@unimore.it
}

\begin{abstract}
Variability is one of the major roadblocks for III-V semiconductors in nanoscale devices, according to the recent International Roadmap for Devices and Systems (IRDS). A particular concern is the detrimental effect of variability of threshold voltage due to channel compositional variations. In this paper, we investigate the impact of this variability source and the effects of scaling on the performance of Dual-Gate-Ultra-ThinBody (DG-UTB) In0.53Ga0.47As MOSFETs. We model mole fraction variations in terms of the Indium content by taking into account the spatial inhomogeneity of the channel and the corresponding bandgap variations, analyzing the effects on threshold voltage variability. We thus define a variability source, i.e., Band Gap Fluctuation (BGF), and we compare the associated variability with the ones from other important sources, namely, Random Dopant Fluctuation (RDF), Work Function Fluctuation (WFF), Body- and Gate- Line Edge Roughness (B-LER and GLER). We then define three corner cases for mole fraction variations to determine worst-case variability. Finally, the impact of scaling on variability is assessed by comparing results for two technology nodes on the linear and saturation threshold voltage, $V_{\mathrm{T}, \text { lin, }} V_{\mathrm{T}, \text { sat, }}$ on-current, IoN, leakage current, IOFF, and linear and saturation sub-threshold slope, $S S$. We find that although scaling has no impact on BGF-induced $V_{T}$ variability, it increases the total $V_{\mathrm{T}, \text { lin }}$ variability as well as that for $I_{\mathrm{ON}}$ and $I_{\mathrm{OFF}}$.
\end{abstract}

Keywords-III-V MOSFETs, Variability, InGaAs, Band Gap Fluctuation, Scaling, IRDS.

\section{INTRODUCTION}

Among the candidates for replacing Silicon in the quest towards extending Moore's law, III-V semiconductors like $\mathrm{In}_{0.53} \mathrm{Ga}_{0.47}$ As [1] stand out for their enhanced mobility and injection velocity [2], [3]. The advent on the market of this technology has been slowed down due to several drawbacks that need to be tackled to successfully meet the roadmap requirements, including the effects of interface and border traps [4], [5], reduced density of states (DOS) [6], as well as potentially larger on-chip statistical variability [7], [8]. The variability of threshold voltage has indeed been described in the recent International Roadmap for Devices and Systems (IRDS) [2] as a major impediment in using III-V materials in ultrascaled devices. Therefore, a careful assessment of variability and its dependence on scaling is important for evaluating the feasibility of InGaAs technology for future nodes.

Variability mainly stems from: $i$ ) discrete distribution of dopants and traps [9]; ii) granularity of the gate metal [10]; iii) roughness of the gate and channel edges [11]. Compared to Silicon, which is affected by variability as well, InGaAs is

\author{
Giovanni Verzellesi \\ Dipartimento di Scienze e Metodi dell'Ingegneria (DISMI) \\ Università degli Studi di Modena e Reggio Emilia \\ 42122, Reggio Emilia (RE), Italy
}

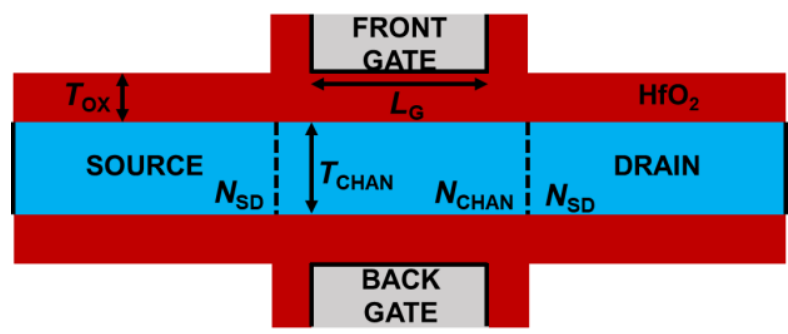

Fig. 1. Schematic representation of the simulated Dual-Gate UTB MOSFET (cross-section).

affected also by variations in the channel composition, i.e., Indium (In) mole fraction $x$. This effect has received little attention in recent literature [12], [13], thus it is crucial to assess its impact on the total variations of key Figures Of Merit (FOMs) in ultra-scaled InGaAs devices.

In this paper, we extend our previous work [14] that analyzed the variability of linear threshold voltage $\left(V_{\mathrm{T}, \mathrm{lin}}\right)$ in scaled InGaAs Dual-Gate Ultra-Thin Body (DG-UTB) including the effect of compositional variations, by investigating also the effect of scaling on the variability on a more comprehensive set of FOMs. These include the threshold voltage in saturation regime, $V_{\mathrm{T}, \mathrm{sat}}$, on-current, $I_{\mathrm{ON}}$, leakage current, $I_{\mathrm{OFF}}$, and subthreshold slope, $S S$ (in both the linear and saturation regimes). The analysis is carried out starting from the calibration of our TCAD simulations through comparison with higher-order models [15]. Scaling effects are considered by evaluating variability at two technology nodes with different physical gate lengths $\left(L_{\mathrm{G}}=15 \mathrm{~nm}\right.$ and $\left.L_{\mathrm{G}}=10.4 \mathrm{~nm}\right)$. The paper is organized as follows. In Section II, we describe the set-up used for calibrating TCAD simulations for both technology nodes. Section III describes the variability modeling framework. Results are shown and commented in Section IV. Finally, in Section V we draw the conclusions of this work.

\section{Simulations Set-UP}

Our simulations are performed by means of the Sentaurus Device simulator [18]. A sketch of the analyzed InGaAs DGUTB MOSFET is shown in Fig. 1. This template device is defined according to the International Technology Roadmap for Semiconductor (ITRS) prescriptions [16]. The two nodes considered in this work have: a) $L_{\mathrm{G}}=15 \mathrm{~nm}, V_{\mathrm{DD}}=0.63 \mathrm{~V}$ and b) $L_{\mathrm{G}}=10.4 \mathrm{~nm}, V_{\mathrm{DD}}=0.59 \mathrm{~V}$. Henceforth, we will refer to these nodes generically as node $\mathrm{A}$ and node $\mathrm{B}$, respectively. This choice is made to avoid confusion with the ITRS 


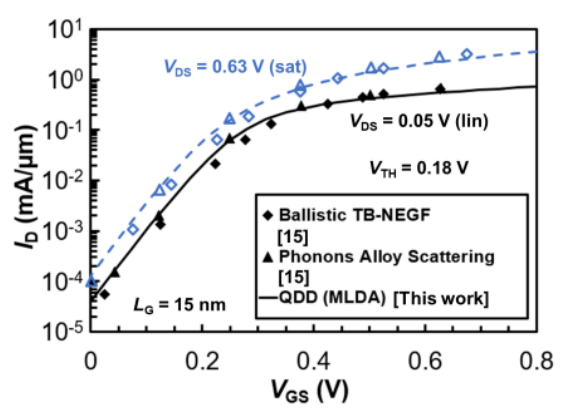

Fig. 2. Calibration of our QDD simulations with higher order models [15] in both the linear (black) and saturation (blue) regimes for node $\mathrm{A}\left(L_{\mathrm{G}}=15 \mathrm{~nm}\right)$.

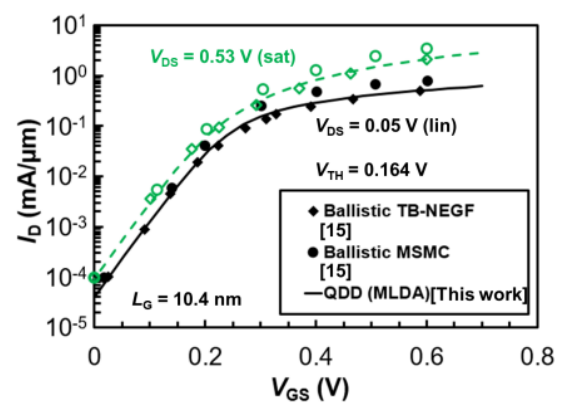

Fig. 3. Calibration of our QDD simulations with higher order models [15] in both the linear (black) and saturation (green) regimes for node $\mathrm{B}\left(L_{\mathrm{G}}=10.4 \mathrm{~nm}\right)$.

terminology used for Si technology nodes (which does not correspond to the physical gate length), and it is discussed more in detail in Section IV.

The simulations are fully-3D quantum-corrected driftdiffusion (QDD). The Modified Local Density Approximation (MLDA) [17] is used to account for quantum confinement effects in the channel. The effects of quasi-ballistic transport are included by means of an effective mobility approach [18]. We calibrated our QDD simulations with higher-order models [15], obtaining the results shown in Figs. 2 and 3 for the two nodes considered in this work. Drain-current $\left(I_{\mathrm{D}}\right)$ vs gate-sourcevoltage $\left(V_{\mathrm{GS}}\right)$ curves in both linear $\left(V_{\mathrm{DS}}=0.05 \mathrm{~V}\right)$ and saturation $\left(V_{\mathrm{DS}}=V_{\mathrm{DD}}\right)$ regimes are shown. The process parameters of the template device for the two nodes are summarized in Tab. I.

\section{VARIABILITY MODELING}

Variability is assessed by means of the statistical Impedance Field Method (IFM) available in Sentaurus Device [18]. Within IFM, the randomness due to the variability sources is accounted for as small perturbations of the contact currents. Therefore, the self-consistent QDD 3D problem needs to be solved only once for the calibrated reference device. The current variations for each variability source are then computed at each bias point in a linearized manner via the Green's function method [19]. A set of varied curves is thus generated, on which standard statistical methods are applied to evaluate the standard deviation of the electrical parameters under consideration (for example $V_{\mathrm{T}, \mathrm{in}}$ ). The sources of statistical variability considered in this work are: Random Dopant Fluctuation (RDF) in both channel and source/drain regions, Work Function Fluctuation (WFF), Bodyand Gate- Line Edge Roughness (B-LER/G-LER), and
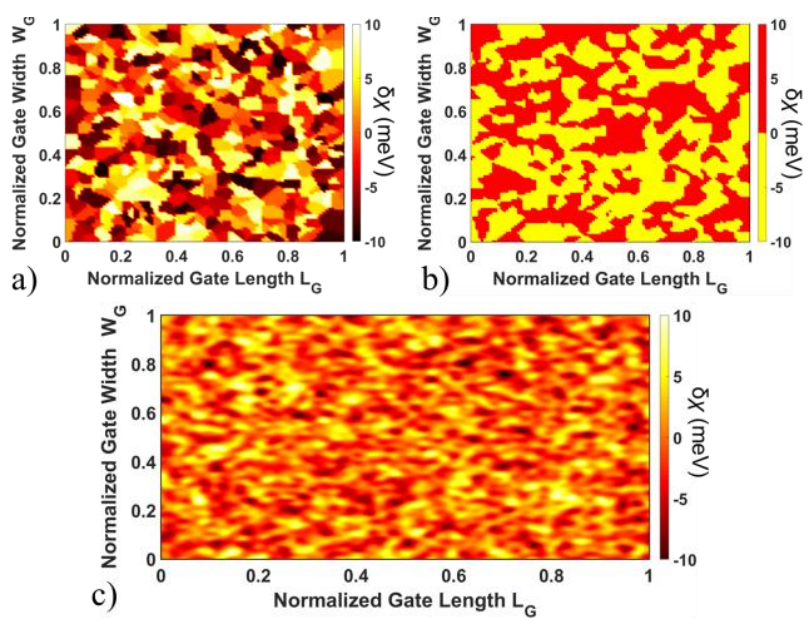

Fig. 4. InGaAs bandgap variation in a $2 \mathrm{D}$ slice of the channel, distributed with Grain tessellation a), b) and with Gaussian approach c). In a) 20 and b), 2 types of grains are considered, respectively. Each grain is characterized by a $E_{\mathrm{G}}$ value. The average normalized grain size in a) and b) is 0.002 . $\delta \chi$ variations are between $\pm 10 \mathrm{meV}$, irrespective of the different tessellation approaches.

TABLE I. NOMINAL VALUES OF PROCESS PARAMETERS FOR THE TWO INGAAS NODES CONSIDERED IN THIS WORK [12], [16].

\begin{tabular}{cc}
\hline \hline Node $\mathbf{A}$ & Node B \\
\hline$L_{\mathrm{G}}=15 \mathrm{~nm}$ & $L_{\mathrm{G}}=10.4 \mathrm{~nm}$ \\
$W_{\mathrm{G}}=26 \mathrm{~nm}$ & $W_{\mathrm{G}}=14 \mathrm{~nm}$ \\
$N_{\mathrm{CHAN}}=10^{17} \mathrm{~cm}^{-3}$ & $N_{\mathrm{CHAN}}=10^{17} \mathrm{~cm}^{-3}$ \\
$N_{\mathrm{S} / \mathrm{D}}=5 \times 10^{19} \mathrm{~cm}^{-3}$ & $N_{\mathrm{S} / \mathrm{D}}=5 \times 10^{19} \mathrm{~cm}^{-3}$ \\
$T_{\mathrm{OX}}=3.8 \mathrm{~nm}$ & $T_{\mathrm{OX}}=3.3 \mathrm{~nm}$ \\
$T_{\mathrm{CHAN}}=7 \mathrm{~nm}$ & $T_{\mathrm{CHAN}}=4 \mathrm{~nm}$ \\
\hline \hline
\end{tabular}

TABLE II. PARAMETER VALUES FOR THE VARIABILITY SOURCES ACCORDING TO ITRS SPECIFICATIONS AND SPECIFIC LITERATURE [10][11].

\begin{tabular}{ccc}
\hline \hline $\begin{array}{c}\text { Band Gap } \\
\text { Fluctuation }\end{array}$ & $\begin{array}{c}\text { Gate- and Body-Line } \\
\text { Edge Roughness }\end{array}$ & $\begin{array}{c}\text { Work Function } \\
\text { Fluctuation }\end{array}$ \\
\hline$\delta \chi=10 \mathrm{meV}$ & $\Delta_{\mathrm{rms}}=1.8 \mathrm{~nm}(15 \mathrm{~nm})$ & $\begin{array}{c}\text { Avg. Grain Size }=5 \\
\mathrm{~nm}\end{array}$ \\
$\Lambda_{\mathrm{BGF}}=300 \mathrm{~nm}$ & $1.2 \mathrm{~nm}(10.4 \mathrm{~nm})$ & $P_{\mathrm{WF} 1}=60 \%$ \\
$\alpha=-1.3$ & & \\
$\delta E_{G}=\alpha \cdot \delta \chi$ & $\Lambda_{\mathrm{LER}}=15.5 \mathrm{~nm}(15 \mathrm{~nm})$ & $P_{\mathrm{WF} 2}=40 \%$
\end{tabular}

Band Gap Fluctuation (BGF). Here we focus on the modeling of BGF, as the details regarding the other four sources have been described elsewhere [12]. The parameters related to the modeling of the variability sources are summarized in Tab. II, and are taken from ITRS and/or specific literature [10], [11]. Specific assumptions need to be made in order to assess the contribution of BGF on the total variability. We define our assumptions by focusing on $V_{\mathrm{T} \text {,lin }}$ variability.

BGF stems from the compositional variations in the InGaAs channel [20], [21]. The spatial inhomogeneity of the Indium content leads to variations $\delta E_{\mathrm{G}}$ of the bandgap. Our modeling approach considers the shifts of both valence and conduction band edges when calculating the total $\delta E_{\mathrm{G}}$ according to the 


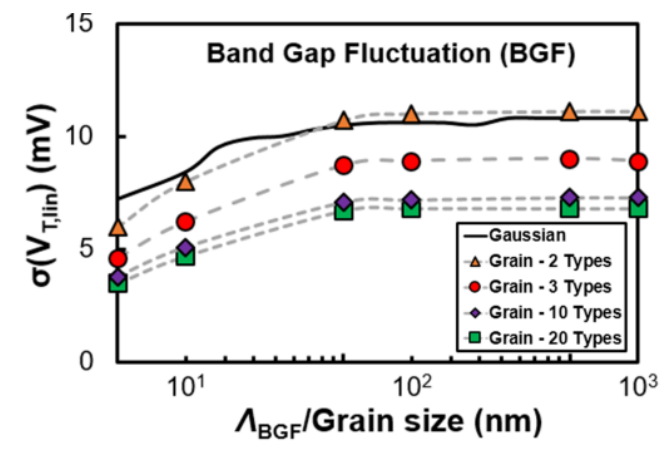

Fig. 5. BGF-induced $V_{\mathrm{T}}$ variability vs. Gaussian correlation length $\left(\Lambda_{\mathrm{BGF}}\right)$ or avg. grain size. Solid line refers to the Gaussian approach, dashed lines to grain tessellation, with different types -2 (orange triangles), 3 (red circles), 10 (purple diamonds) and 20 (green squares). For each case, $\delta \chi=10 \mathrm{meV}$ (see Tab. I).
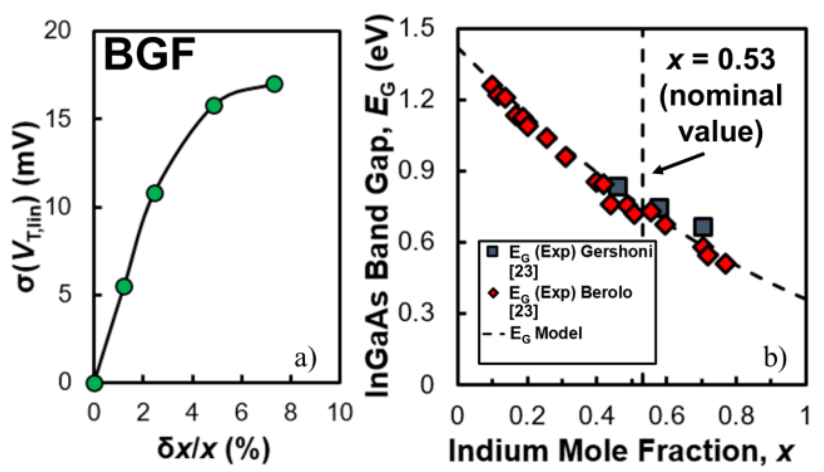

Fig. 6. (a) BGF-induced $V_{\mathrm{T}, \text { lin }}$ variability dependence on relative mole fraction variation, $\delta x / x$. (b) InGaAs Band Gap, $E_{\mathrm{G}}$ vs. $x$ model used in the simulator (dashed line) compared with experimental data from ref. [23] (symbols).

simple model in Tab. II, relating it to electron affinity variations $\delta \chi$ [22]. The value of $10 \mathrm{meV}$ for $\delta \chi$ is chosen in accordance to the data found in [21], by considering the expected experimental range of mole fraction variations. The inhomogeneity due to the In content variations is modeled via a granular approach similar to that adopted for WFF to account for metal granularity [10], [12]. Specifically, the channel volume is divided into "grains" of different types and sizes each associated with a different bandgap value $\left(E_{\mathrm{G}}\right)$. The grain size is randomized around an average value. This procedure is visually exemplified in Figs. 4a) and b), showing the representation for two different possible tessellations of the channel considering 20 and 2 grain types, respectively. Note that in Fig. 4 dimensions have been normalized for simplicity, as the purpose here is to give a visual representation of the modeling approach. A computationally more effective solution with respect to the finite-size grain tessellation is to adopt a continuous random profile that approximates the discrete approach. The visual representation is shown in Fig. 4c). The continuous profile is generated by using a Fourier approach with a Gaussian covariance function (hereafter referred to as Gaussian approach) [18], and is characterized by a correlation length, $\Lambda_{\mathrm{BGF}}$ (analogous to the avg. grain size) and the peak $E_{\mathrm{G}}$ variation value, $\delta E_{\mathrm{G}}$, see Tab. II. To highlight the effects in terms of BGF-induced variability of the different tessellation approaches, we consider a varying grain size (or correlation length $\Lambda_{\mathrm{BGF}}$ for the Gaussian approach) and

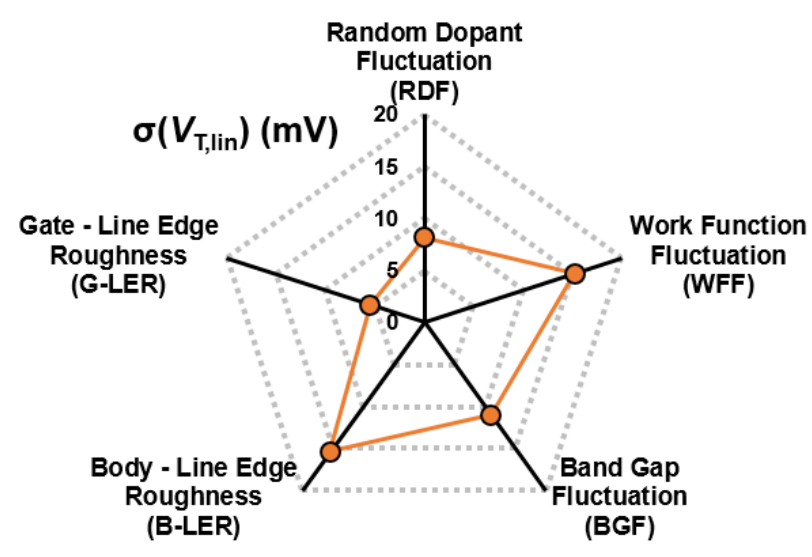

Fig. 7. $V_{\mathrm{T}, \text { lin }}$ variability, $\sigma\left(V_{\mathrm{T}, \text { lin }}\right)$, for the five sources considered in our simulations. WFF and B-LER are the dominant sources, while BGF is greater than RDF and G-LER (the latter being the least impacting source).

evaluate the $V_{\mathrm{T}, \text { lin }}$ variability. The results are shown in Fig. 5, where the grain size (or $\Lambda_{\mathrm{BGF}}$ ) is varied between $5 \mathrm{~nm}$ and $1 \mu \mathrm{m}$ considering up to 20 different grain types (different symbols correspond to different number of grain types). As shown in Fig. 5, the Gaussian approach well approximates the grain tessellations when considering two possible grain types only. This is due to the fact that a larger number of possible grain types reduces the BGF-induced $\sigma\left(V_{\mathrm{T}, \text { lin }}\right)$. In all cases, $\sigma\left(V_{\mathrm{T}, \text { lin }}\right)$ saturates at high $\Lambda_{\mathrm{BGF}}$ or avg. grain size; therefore, by adopting a Gaussian approach with $\Lambda_{\mathrm{BGF}}=300 \mathrm{~nm}$, see Fig. 5 , we can perform a worst-case estimation of the BGF-induced $\sigma\left(V_{\mathrm{T}, \text { lin }}\right)$. Note that the bandgap variations can be directly translated into mole fraction variations thanks to their physical interplay. Fig. 6(a) explains this relation, by showing the variation of $\sigma\left(V_{\mathrm{T}, \text { lin }}\right)$ induced by BGF with varying In mole fraction with respect to the nominal value $x=0.53$ (i.e., relative variation $\delta x / x$ ). Fig. 6(b) shows with a dashed line the $E_{\mathrm{G}}$ vs. $x$ model implemented in the simulator, which fits the experimental data (symbols) as taken from [23] and references therein.

\section{RESULTS AND DISCUSSION}

In this section, we first discuss the results on the $V_{\mathrm{T}, \text { lin }}$ variability considering all the five sources described in the previous section. Then, we make projections on the total $V_{\mathrm{T} \text {,lin }}$ variability based on the variations of the mole fraction $x$ taking into account the statistical distribution of $x$ (for $\operatorname{In}_{0.53} \mathrm{Ga}_{0.47} \mathrm{As}$ ) from experiments reported in the literature. We then define three possible corner cases (CCs) that allow considering the sideeffects of channel composition variations on other variability sources and on the nominal $V_{\mathrm{T}, \text { lin. }}$. Finally, to assess the effects of scaling on variability for the full set of FOMs defined in the Introduction, we compare the results of the variability analysis on all the five sources for both node A and B.

\section{A. $B G F V_{\mathrm{T}, \text { lin }}$ variability (node $A$ )}

In Fig. 7, we show the variability of the five different sources on the $V_{\mathrm{T}, \text { lin }}$ (defined as $\sigma\left(V_{\mathrm{T}, \text { lin }}\right)$ ), comparing their relative contributions. According to Fig. 7, BGF - modeled following the approach described in Section II - is the third most impacting source after WFF and B-LER, which have 


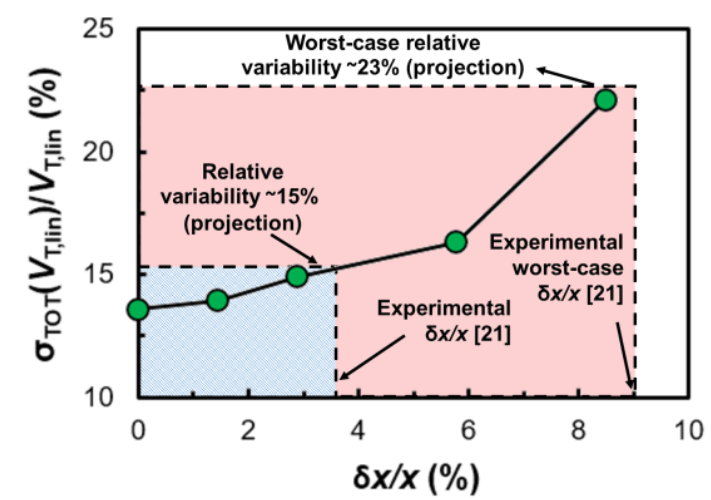

Fig. 8. Simulated $\sigma_{\mathrm{TOт}}\left(V_{\mathrm{T}, \text { lin }}\right) / V_{\mathrm{T}}$,lin relative variation vs. relative mole fraction variation, $\delta x / x$. The blue box indicates a "safe region" in which the projected $\sigma_{\mathrm{TOT}}\left(V_{\mathrm{T}, \text { lin }}\right) / V_{\mathrm{T}, \text { lin }}$ is $\sim 15 \%$, extracted from the experimental range of mole fraction variation found in [21]. The red box indicates the worst-case total variability with the corresponding worst-case $\delta x / x$, also from [21].

comparable effects [12], with a standard deviation of $\sim 15 \mathrm{mV}$. It is worth mentioning that the $\sigma\left(V_{\mathrm{T}, \text { lin }}\right)$ value due to $\mathrm{BGF}$ in Fig. 6 is calculated by considering the average mole fraction variations that are attained in the experiments [21]. Although this result gives the positive indication that BGF should not be the first concern regarding $V_{\mathrm{T} \text {,in }}$ variability, it is instructive to analyze more in detail the impact of BGF-induced $\sigma\left(V_{\mathrm{T}, \text { lin }}\right)$. This is done by evaluating the relative BGF-induced $\sigma\left(V_{\mathrm{T}, \text { in }}\right)$ (normalized to the nominal threshold voltage) at different mole fraction variations, see Fig. 6a), and then by computing the total $V_{\mathrm{T} \text {,in }}$ variability as the square sum of the individual contributions from all sources (i.e., assumed to be independent). The results are shown in Fig. 8, with two highlighted regions indicating the variability projections evaluated starting from the statistical distribution (for a nominal value of 0.53 ) of the experimental $\delta x / x$ values taken from [21]. The blue box region indicates a "safe region" for which the projected $\sigma_{\mathrm{TOT}}\left(V_{\mathrm{T}, \text { lin }}\right) / V_{\mathrm{T}, \text { lin }}$ is $\sim 15 \%$, corresponding to the average mole fraction variations as reported in [21]. The red box instead shows the projected worst-case $\sigma_{\mathrm{TOT}}\left(V_{\mathrm{T}, \text { lin }}\right) / V_{\mathrm{T}, \text { lin }}$ of $\sim 23 \%$, corresponding to the worst-case $\delta x / x$ of about $9 \%$ as reported in [21]. These projections on $\sigma_{\mathrm{TOT}}\left(V_{\mathrm{T}, \text { lin }}\right) / V_{\mathrm{T}, \text { lin }}$ may serve as a reference for technologists to: $i$ ) target appropriate $x$ control to attain reasonable variability, or ii) get worst-case total variability projections of their fabricated devices (for a given expected $\delta x / x)$.

Channel composition variations not only cause bandgap to vary but also alter other material properties like the dielectric constant and the electron effective mass. Therefore, it is reasonable to expect side-effects on the electrostatic behavior of the InGaAs device and on the other variability sources. To investigate these issues, we perform a sensitivity analysis (following the terminology in [12]) - by considering three possible CCs. These CCs are identified by considering the In mole fraction variations or, equivalently, the corresponding BGF-induced $\sigma\left(V_{\mathrm{T}, \text { lin }}\right)$ as shown in Fig. 6a). The first $\mathrm{CC}$ under study, labeled $\mathrm{CC} 1$, is defined in such a way that the BGF variability is numerically equivalent to the G-LER (i.e., the least impacting source, see Fig. 7). The third corner case, CC3,

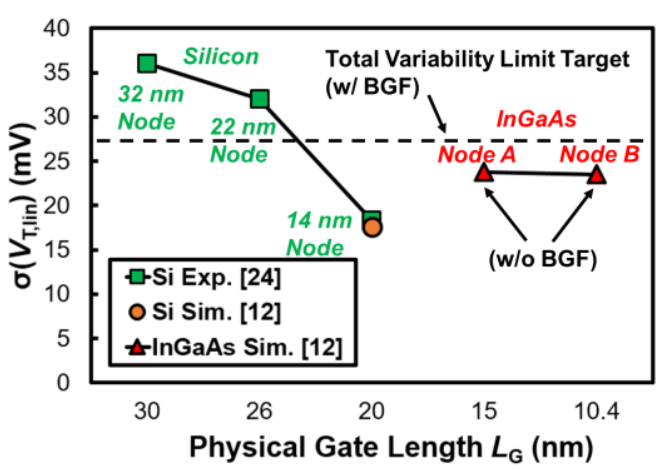

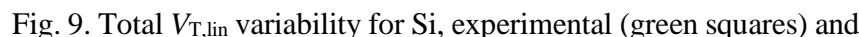
simulation (orange circle) and for InGaAs, sim. only (red triangles) for different technology nodes (following the terminology for $\mathrm{Si}$ ). The labels near each Si point indicate the corresponding technology node for each physical $L_{\mathrm{G}}$. A target $V_{\mathrm{T}, \text { lin }}$ variability limit is defined for InGaAs node A imposing a maximum tolerable BGF value for the corner case $\mathrm{CC} 2$

is obtained in a similar way to $\mathrm{CC} 1$ by setting BGF-induced $\sigma\left(V_{\mathrm{T}, \text { in }}\right)$ equal to the one induced by either WFF or B-LER (i.e., the most impacting sources). The second corner case, CC2, is obtained by imposing an upper limit on the total $\sigma\left(V_{\mathrm{T}, \text { lin }}\right)$ (calculated as the square sum of the contributions due to the individual sources). The limit is set to $27 \mathrm{mV}$ considering that this value: $i$ ) lies in between the experimental $\sigma_{\mathrm{TOT}}\left(V_{\mathrm{T}, \mathrm{lin}}\right)$ of the $22 \mathrm{~nm}$ and $14 \mathrm{~nm}$ nodes for Si technology [24], see Fig. 9; ii) guarantees a safe margin when accounting also for BGFinduced variability in the $\sigma_{\mathrm{TOT}}\left(V_{\mathrm{T}, \text { lin }}\right)$ for InGaAs. We mention the fact that although no significantly large experimental $V_{T}$ variability dataset for $\operatorname{In}_{0.53} G a_{0.47} A s$ scaled devices can be found in the literature, the dependability of our simulation approach for variability is supported by the agreement of simulations with experimental Si variability data [12], Fig. 9.

The three CCs defined above are effectively used to define six varied devices, each with a different nominal mole fraction value. These devices differ from the nominal calibrated device by their mole fraction, obtained by considering that each $\mathrm{CC}$ identifies both a maximum and minimum $x$ value ( $x$ variations are considered as both increments and decrements from the nominal value). The variability analysis is then performed separately on each of these six different devices. Note that in this context, BGF is not accounted for directly, but as a consequence of the variation in the nominal mole fraction when defining the six different devices.

We now analyze the variability for each of the six devices in terms of $\sigma\left(V_{\mathrm{T}, \text { in }}\right)$ for RDF, WFF, B-LER and G-LER. Results are collected in Fig. 10, for the maximum [Fig. 10a)] and the minimum [Fig. 10b)] In mole fraction values for the three CCs under investigation. The nominal case is included as well for a clear comparison. Fig. 10 shows that the variability sources are negligibly affected by $x$ variations, with the exception of $\mathrm{B}$ LER. The variation of B-LER-induced $\sigma\left(V_{\mathrm{T}, \text { lin }}\right)$ is particularly evident in the case of $\mathrm{CC} 3$, for both maximum and minimum $x$ values. Because of this variation in B-LER-induced $\sigma\left(V_{\mathrm{T}, \mathrm{lin}}\right)$, the corresponding $x$ variations could in turn alter the relative role of this source with respect to the other dominant source in the nominal device, i.e., WFF. This clearly occurs for increasing $x$ 

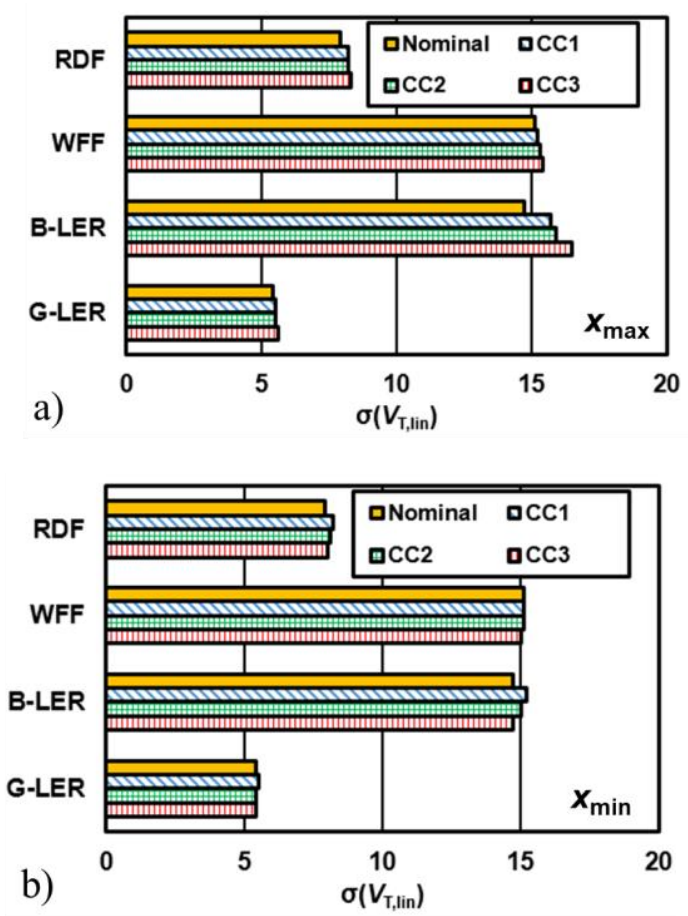

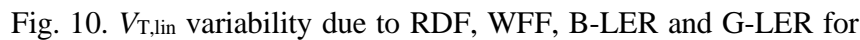
the minimum (a) and maximum (b) In mole fractions variations due to the three corner cases analyzed (from top to bottom): nominal case (yellow bins), $\mathrm{CC} 1$ (blue striped), CC2 (green squared bins) and CC3 (red lined bins).

values, as shown in Fig. 11, where $\sigma\left(V_{\mathrm{T}, \text { lin }}\right)$ is plotted against $x$ for B-LER and WFF. The main finding from Fig. 11 is that, at increasing $x$, B-LER takes the dominant role with respect to WFF. Similar considerations on the side-effects of varying mole fraction apply to the nominal $V_{\mathrm{T}, \text { lin, }}$, which is of course different for each device realization. The variation of the nominal $V_{\mathrm{T}, \text { lin }}$ is shown in Fig. 12, as well as the variation of $\sigma_{\mathrm{TOT}}\left(V_{\mathrm{T}, \mathrm{lin}}\right)$ (not including BGF). Fig. 12 clearly indicates that the side-effects of BGF are significant, both on $V_{\mathrm{T}, \text { lin }}$ and $\sigma_{\mathrm{TOT}}\left(V_{\mathrm{T}, \text { lin }}\right)$. Therefore, these findings should be taken into consideration in the optimization of process conditions under the variability-limited scaling context forecasted by IRDS for InGaAs technology.

\section{B. Comparison with node $B$}

The modeling framework for BGF discussed in Section IV.A is exploited to assess the variability for node B as well. The device calibration curves and process parameters are included in Fig. 3 and Tab. I, respectively. As discussed in the Introduction, we carry on the variability analysis for a more extended set of FOMs, i.e., $V_{\mathrm{T} \text {,lin, }} \mathrm{V}_{\mathrm{T} \text {,sat }}, I_{\mathrm{ON}}, I_{\mathrm{OFF}}$ and $S S$ (linear and saturation regimes). In addition, we compare the results with node $\mathrm{A}$ to assess the overall effect of scaling. The variability for each FOM has been normalized to the respective nominal value for fair comparison between the two nodes. Figs. 13-15 show the results of the analysis for: a) $V_{\mathrm{T} \text {,lin }}$ and $V_{\mathrm{T} \text {,sat }}$, Fig. 13 ; b) $I_{\mathrm{ON}}$ and $I_{\mathrm{OFF}}$, Fig. 14 and; c) $S S$ (in both the linear and saturation regime), Fig. 15. From Fig. 13, it is possible to notice that all

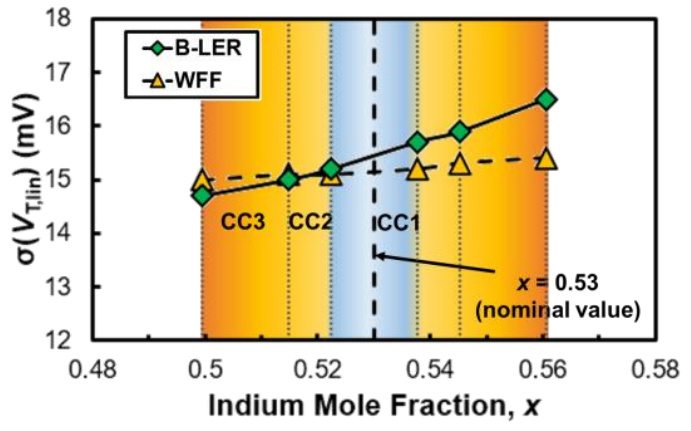

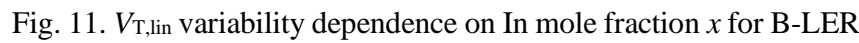
(green diamonds) and WFF (yellow triangles). The three corner cases are highlighted with colored areas in the background.

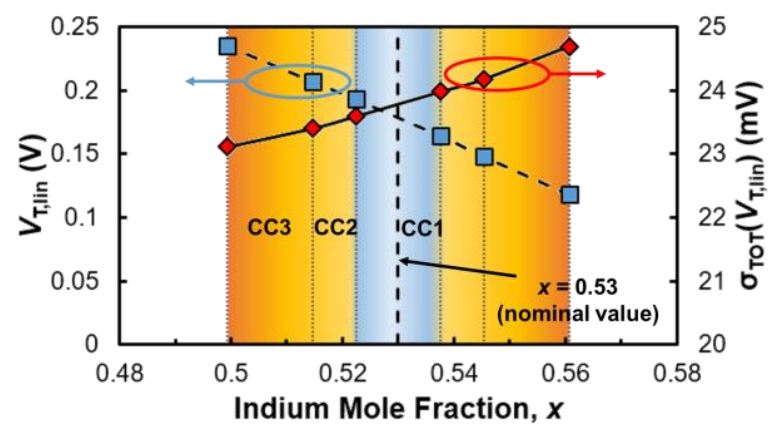

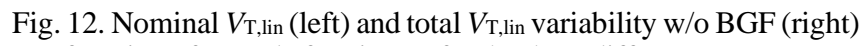
as a function of In mole fraction, $x$, for the three different corner cases, highlighted with colored areas in the background.

the variability sources are affected by scaling, with the notable exception of BGF. While B-LER and G-LER are reduced thanks to the decrease of their characteristic parameters according to ITRS prescriptions [16], we find that RDF and WFF increase with scaling. This problem can be mitigated by adopting specific engineering strategies, outlined here briefly. In general, RDF can be reduced by reducing doping in the channel [25]; WFF instead, can be reduced by improving the granularity of the metal gate via gate-last processes or by using more amorphous materials [26], [27]; finally, LER can be effectively limited with improved lithographic techniques [like Extreme UV lithography (EUV)], or by relying on epitaxy rather than lithography for the definition of critical dimensions [28]. In perspective, if all the variability sources are reduced, BGF impact could become more severe in ultra-scaled nodes unless technological solutions for limiting $x$ variations are devised. Similar considerations apply to $V_{\mathrm{T} \text {,sat }}$ variability [Fig. $13 \mathrm{~b})$ ], as it is clear that the relative role of the variability sources is the same as for $V_{\mathrm{T}, \text { lin }}$.

Fig. 14 shows the impact of the various variability sources on a) $I_{\mathrm{ON}}$ and b) $I_{\mathrm{OFF}}$, respectively. Interestingly, $\sigma\left(I_{\mathrm{ON}}\right)$ is found to increase from node A to B for all sources, with the exception of G-LER. Particularly, RDF-induced $\sigma\left(I_{\mathrm{ON}}\right)$ dramatically increases, becoming the only dominant variability source for node B. Fig. $14 \mathrm{~b})$ shows that $\sigma\left(I_{\mathrm{OFF}}\right)$ is clearly dominated by BLER for node $\mathrm{A}$, whereas in node $\mathrm{B}$ WFF becomes comparable to B-LER. We note that $\sigma\left(I_{\mathrm{OFF}}\right)$ and $\sigma\left(V_{\mathrm{T} \text {,sat }}\right)$ are strongly correlated, as expected from their physical interdependence. 


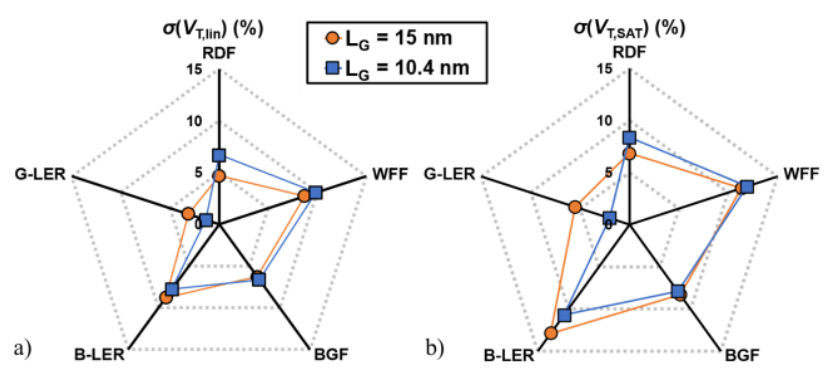

Fig. 13. Relative Threshold voltage $V_{\mathrm{T}}$ variability, $\sigma\left(V_{\mathrm{T}}\right)$, for the five sources, in both the linear a) and saturation b) regime. Orange circles (blue squares) correspond to the $15 \mathrm{~nm}(10.4 \mathrm{~nm})$ node.

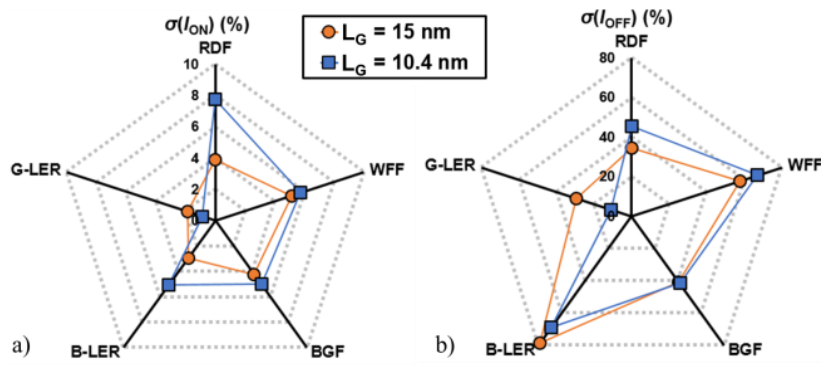

Fig. 14. Relative $I_{\mathrm{ON}}$ a) and $\left.I_{\mathrm{OFF}} \mathrm{b}\right)$ variability, $\sigma\left(I_{\mathrm{ON}}\right), \sigma\left(I_{\mathrm{OFF}}\right)$, for the five sources. Orange circles (blue squares) correspond to the $15 \mathrm{~nm}$ $(10.4 \mathrm{~nm})$ node.

Finally, Fig. 15 shows that from what concerns $S S-$ in both regimes - the variability due to each of the five sources has a negligible impact. The dominant source in this case is B-LER, whose effect is further reduced when scaling from node A to B.

Finally, Tab. III reports the total relative variability for each of the FOMs, along with their corresponding absolute values. The total variability is given by the square sum of the contributions due to all the five variability sources (RDF, WFF, BGF, B-LER, and G-LER). The results included in Tab. III give different trends for the total relative variability of the FOMs. In fact, while $\sigma_{\mathrm{TOT}}\left(V_{\mathrm{T}, \mathrm{lin}}\right)$, $\sigma_{\mathrm{TOT}}\left(I_{\mathrm{ON}}\right)$ and $\sigma_{\mathrm{TOT}}\left(I_{\mathrm{OFF}}\right)$ increase with scaling, the variability for the other FOMs reduces. It is also important to notice that for a nominal $I_{\mathrm{OFF}}=100 \mathrm{nA} / \mu \mathrm{m}$, our simulations predict that the total $I_{\mathrm{OFF}}$ variability, $\sigma_{\mathrm{TOT}}\left(I_{\mathrm{OFF}}\right)$, exceeds this value at both nodes, mainly due to the effect of BLER, indicating that a poor control over the variability of this parameter is currently attained with the DG-UTB technology, requiring further improvements in the lithography resolution.

\section{CONCLUSIONS}

In this work, we assess the role of mole fraction variations in nanoscale $\operatorname{In}_{\mathrm{x}} \mathrm{Ga}_{1-\mathrm{x}}$ As MOSFETs as well as the effects of scaling. We first define a modeling approach to assess the Band Gap Fluctuation (BGF) effect on the variability of the threshold voltage. We then make projections on the BGF variability impact on experimental mole fraction distributions to extract the total relative $V_{\mathrm{T} \text {,lin }}$ variability. These projections can be a useful reference for technologists to target appropriate $x$ control to attain reasonable variability and/or to get worst-case total variability projections for fabricated devices. Moreover, we identify three corner cases to evaluate the impact of mole fraction variations on other variability sources as

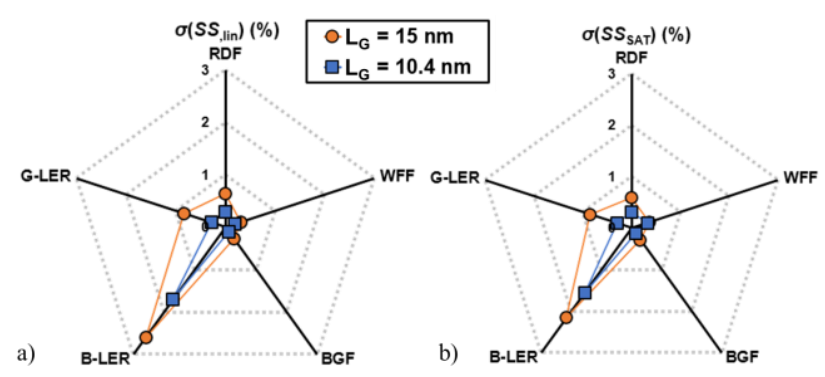

Fig. 15. Relative subthreshold slope variability, $\sigma(S S)$, for the five sources, in both a) the linear and b) saturation regime. Orange circles (blue squares) correspond to the $15 \mathrm{~nm}(10.4 \mathrm{~nm})$ node.

TABLE III. TOTAL VARIABILITY FOR THE FOUR FOMS FOR INGAAS NODES A AND B.

\begin{tabular}{ccccc}
\hline \hline Variability & \multicolumn{2}{c}{ Node A } & \multicolumn{2}{c}{ Node B } \\
\hline & lin & sat & lin & sat \\
$\sigma_{\mathrm{TOT}}\left(V_{\mathrm{T}}\right)(\%)$ & 15.0 & 21.0 & 15.8 & 19.8 \\
$(\mathrm{mV})$ & 26.2 & 28.2 & 25.9 & 27.4 \\
$\sigma_{\mathrm{TOT}}\left(I_{\mathrm{ON}}\right)(\%)$ & $/ /$ & 8.5 & $/ /$ & 12.0 \\
$(\mu \mathrm{A} / \mu \mathrm{m})$ & $/ /$ & 192.9 & $/ /$ & 284.6 \\
$\sigma_{\mathrm{TOT}}\left(I_{\mathrm{OFF}}\right)(\%)$ & $/ /$ & 110.1 & $/ /$ & 114.6 \\
$(\mathrm{nA} / \mu \mathrm{m})$ & $/ /$ & 145.7 & $/ /$ & 108.3 \\
$\sigma_{\mathrm{TOT}}(S S)(\%)$ & 2.8 & 2.4 & 1.8 & 1.6 \\
$(\mathrm{mV} / \mathrm{dec})$ & 2.0 & 1.7 & 1.2 & 1.1 \\
\hline \hline
\end{tabular}

well, finding a non-negligible effect on Body- Line Edge Roughness (B-LER) only. This result indicates that the relative role of B-LER, with respect to other sources, can be altered by mole fraction variations. We then extend the variability analysis to other important FOMs (i.e., $I_{\mathrm{ON}}, I_{\mathrm{OFF}}$ and $S S$ ) to assess the effect of scaling by considering two technology nodes with different physical gate length (15 and $10.4 \mathrm{~nm}$, respectively). We find that for the BGF-induced $V_{\mathrm{T}, \text { lin }}$ variability scaling has no appreciable impact, although the total relative $\sigma\left(V_{\mathrm{T}, \text { lin }}\right)$ increases, as well as $\sigma\left(V_{\mathrm{T}, \mathrm{sat}}\right), \sigma\left(I_{\mathrm{ON}}\right)$ and $\sigma\left(I_{\mathrm{OFF}}\right)$. $S S$ variability is found to be negligible in both linear and saturation regimes, with a slight reduction observed on the more scaled node. Finally, our simulations indicate that the total $I_{\mathrm{OFF}}$ variability is larger than the nominal value for both nodes, indicating poor variability control of the DG-UTB technology on this parameter.

\section{ACKNOWLEDGMENT}

The authors wish to thank Luca Selmi for fruitful discussion. This research has received funding from the European Commission's Seventh Framework Programme (FP7/20072013) under grant agreement III-V-MOS Project n619326.

\section{REFERENCES}

[1] J. A. del Alamo, "Nanometre-scale electronics with III-V compound semiconductors," Nature, vol. 479, no. 7373, pp. 317-323, Nov. 2011.

[2] IEEE, "International Roadmap for Devices and Systems," 2016.

[3] L. Selmi et al., "Modelling nanoscale n-MOSFETs with III-V compound semiconductor channels: From advanced models for band structures, electrostatics and transport to TCAD," in 2017 IEEE International Electron Devices Meeting (IEDM), 2017, p. 13.4.113.4.4. 
[4] A. Alian et al., "Impact of the channel thickness on the performance of ultrathin InGaAs channel MOSFET devices," Tech. Dig. - Int. Electron Devices Meet. IEDM, pp. 437-440, 2013.

[5] P. Pavan et al., "The impact of interface and border traps on currentvoltage, capacitance-voltage, and split-CV mobility measurements in InGaAs MOSFETs," Phys. Status Solidi Appl. Mater. Sci., vol. 214, no. 3, 2017.

[6] M. V. Fischetti et al., "Simulation of Electron Transport in HighMobility MOSFETs: Density of States Bottleneck and Source Starvation," in 2007 IEEE International Electron Devices Meeting, 2007, pp. 109-112.

[7] N. Zagni, F. M. Puglisi, G. Verzellesi, and P. Pavan, "Combined variability/sensitivity analysis in III-V and silicon FETs for future technological nodes," in IEEE International Reliability Physics Symposium Proceedings, 2017.

[8] N. Zagni, F. M. Puglisi, P. Pavan, and G. Verzellesi, "Random dopant fluctuation variability in scaled InGaAs dual-gate ultra-thin body MOSFETs: Source and drain doping effect," in IEEE International Integrated Reliability Workshop Final Report, 2018, vol. 2017Octob, pp. 1-4.

[9] X. Wang, S. Roy, A. R. Brown, and A. Asenov, "Impact of STI on Statistical Variability and Reliability of Decananometer MOSFETs," IEEE Electron Device Lett., vol. 32, no. 4, pp. 479-481, Apr. 2011.

[10] A. R. Brown, N. M. Idris, J. R. Watling, and A. Asenov, "Impact of metal gate granularity on threshold voltage variability: A full-scale three-dimensional statistical simulation study," IEEE Electron Device Lett., vol. 31, no. 11, pp. 1199-1201, 2010.

[11] A. Asenov, S. Kaya, and A. R. Brown, "Intrinsic parameter fluctuations in decananometer mosfets introduced by gate line edge roughness," IEEE Trans. Electron Devices, vol. 50, no. 5, pp. 1254 1260, May 2003.

[12] N. Zagni, F. M. Puglisi, G. Verzellesi, and P. Pavan, "Threshold Voltage Statistical Variability and Its Sensitivity to Critical Geometrical Parameters in Ultrascaled InGaAs and Silicon FETs," IEEE Trans. Electron Devices, vol. 64, no. 11, pp. 4607-4614, Nov. 2017.

[13] N. Seoane, G. Indalecio, D. Nagy, K. Kalna, and A. J. GarciaLoureiro, "Impact of Cross-Sectional Shape on 10-nm Gate Length InGaAs FinFET Performance and Variability," IEEE Trans. Electron Devices, vol. 65, no. 2, pp. 456-462, Feb. 2018.

[14] N. Zagni, F. M. Puglisi, P. Pavan, and G. Verzellesi, "On the impact of channel compositional variations on total threshold voltage variability in nanoscale InGaAs MOSFETs," in 2018 Joint International EUROSOI Workshop and International Conference on Ultimate Integration on Silicon, EUROSOI-ULIS 2018, 2018, vol. 2018-Janua, pp. 1-4.

[15] M. Rau et al., "Performance Projection of III-V Ultra-Thin-Body ,
FinFET, and Nanowire MOSFETs for two Next- Generation Technology Nodes," pp. 758-761, 2016.

[16] "2013 ITRS - International Technology Roadmap for Semiconductors," 2013. [Online]. Available: http://www.itrs2.net/2013-itrs.html. [Accessed: 09-Jun-2018].

[17] G. Paasch and H. Übensee, "A Modified Local Density Approximation. Electron Density in Inversion Layers," Phys. status solidi, vol. 113, no. 1, pp. 165-178, Sep. 1982

[18] Synopsys, "Sentaurus L-2016.03." 2016.

[19] K. El Sayed, E. Lyumkis, and A. Wettstein, "Modeling Statistical Variability with the Impedance Field Method A systematic comparison between the Impedance Field and the "Atomistic Method," in Simulation of Semiconductor Processes and Devices (SISPAD), 2012 International Conference on, 2012, pp. 205-208.

[20] N. Seoane, A. J. García-Loureiro, K. Kalna, and A. Asenov, "Current variations in PHEMTS introduced by channel composition fluctuations," J. Phys. Conf. Ser., vol. 38, no. 1, pp. 212-215, May 2006

[21] P. R. Pinsukanjana et al., "InGaAs composition monitoring for production MBE by in situ optical-based flux monitor (OFM)," $J$. Cryst. Growth, vol. 251, no. 1-4, pp. 124-129, 2003.

[22] IOFFE, "NSM Archive - Physical properties of Gallium Indium Arsenide (GaInAs)." [Online]. Available: http://www.ioffe.ru/SVA/NSM/Semicond/GaInAs/. [Accessed: 09Jun-2018].

[23] J. Kim and M. V. Fischetti, "Electronic band structure calculations for biaxially strained $\mathrm{Si}, \mathrm{Ge}$, and III-V semiconductors," J. Appl. Phys., vol. 108, no. 1, p. 013710, Jul. 2010.

[24] S. Natarajan et al., "A 14nm Logic Technology Featuring 2 nd Generation FinFET Interconnects, Self-Aligned Double Patterning and a $0.0588 \mathrm{~m} 2$ SRAM cell size," IEDM, IEEE Tech. Dig. - Int. Electron Devices Meet., p. 3.7.1-3.7.3, 2014.

[25] X. Wang, A. R. Brown, B. Cheng, A. Asenov, Binjie Cheng, and A. Asenov, "Statistical variability and reliability in nanoscale FinFETs," Tech. Dig. - Int. Electron Devices Meet. IEDM, pp. 103-106, Dec. 2011.

[26] J. Franco et al., "Gate stack thermal stability and PBTI reliability challenges for 3D sequential integration: Demonstration of a suitable gate stack for top and bottom tier nMOS," in 2017 IEEE International Reliability Physics Symposium (IRPS), 2017, p. 2B-3.1-2B-3.5.

[27] T. Matsukawa et al., "Suppressing Vt and Gm variability of FinFETs using amorphous metal gates for $14 \mathrm{~nm}$ and beyond," in 2012 International Electron Devices Meeting, 2012, p. 8.2.1-8.2.4.

[28] L. Czornomaz et al., "Scalability of ultra-thin-body and BOX InGaAs MOSFETs on silicon," in 2013 Proceedings of the European SolidState Device Research Conference (ESSDERC), 2013, pp. 143-146. 